\title{
Throughput analysis for coexisting IEEE 802.15.4 and 802.11 networks under unsaturated traffic
}

\author{
Phuong Luong, Tri Minh Nguyen and Long Bao Le*
}

\begin{abstract}
Wireless personal area networks (WPAN) and wireless local area networks (WLAN) based on IEEE 802.15.4 and 802.11 standards operate in overlapping unlicensed frequency bands; therefore, they create harmful interference for each other if deployed in the same geographical area. There has been various performance analysis of the media access control (MAC) protocols for both networks individually. However, the coexistence performance of the two networks is less well-understood, which has been mainly studied via computer simulation. In this paper, we attempt to close this gap by developing a comprehensive mathematical model to evaluate the throughput performance of the carrier sense multiple access with collision avoidance (CSMA/CA) protocols of coexisting 802.15.4 WPAN and 802.11 WLAN. Specifically, we consider two coexistence scenarios, called symmetric and asymmetric scenarios. In the symmetric scenario, wireless nodes in both networks can sense one another while in the asymmetric scenario, only WPAN nodes can sense active WLAN nodes but WLAN nodes cannot sense the transmissions from WPAN nodes. The proposed models effectively resolve the major challenge arising from the fact that the MAC protocols in the two networks operate in different time scales. In addition, we explicitly capture detailed operations and interactions of the underlying MAC protocols. We then propose to employ the developed models for channel allocation to achieve fair throughput sharing among WPAN nodes. Numerical results confirm the excellent accuracy of the proposed models and their usefulness for performance evaluation and design of the heterogeneous network.
\end{abstract}

Keywords: Coexistence, IEEE 802.15.4, IEEE 802.11, WLAN, ZigBee, Markov chain, Throughput performance, CSMA/CA MAC protocol, Fairness

\section{Introduction}

The IEEE 802.15.4-based wireless personal area network (WPAN) has been widely employed in many applications, thanks to its low-cost and low-power characteristics. IEEE $802.11 \mathrm{~b} / \mathrm{g}$ wireless local area network (WLAN) is another standard which has seen tremendous success with widespread adoption worldwide. However, both IEEE 802.15.4 WPAN and IEEE 802.11b/g WLAN operate in the overlapping 2.4-GHz unlicensed ISM spectrum. As a result, the WPAN performance can be severely impacted by transmissions from the higher-power WLAN if they are located in the same geographical area. Study of the coexistence performance between these WPAN and WLAN and development of enhanced solutions for

${ }^{*}$ Correspondence: long.le@emt.inrs.ca

INRS-EMT, University of Quebec, Montreal, QC, Canada these networks are important research problems. Given ZigBee is one of the most popular standards based on IEEE 802.15.4, we adopt the implicit custom in the literature where we refer to IEEE 802.15.4 WPAN and ZigBee network interchangeably in this paper. Because of the crowded unlicensed ISM spectrum, it would be every challenging to configure all IEEE 802.11 WLAN and IEEE 802.15.4 ZigBee networks to operate on orthogonal frequency bands.

There have been several measurements reporting that the IEEE 802.15.4-based WPAN can experience high packet loss as they operate on the same channel and suffer from co-channel interference from IEEE 802.11 WLAN [1-5]. Interactions between these two radio technologies were investigated when the distance between ZigBee and WLAN nodes was varied in $[1,2]$. In [1], the authors confirmed that the WLAN nodes still perform

\section{Springer}

(c) 2016 Luong et al. Open Access This article is distributed under the terms of the Creative Commons Attribution 4.0 International License (http://creativecommons.org/licenses/by/4.0/), which permits unrestricted use, distribution, and reproduction in any medium, provided you give appropriate credit to the original author(s) and the source, provide a link to the Creative Commons license, and indicate if changes were made. 
backoff during the ZigBee transmissions if the WLAN and ZigBee nodes are sufficiently separated. In addition, it was shown that WLAN can suffer from the harmful interference when coexisting with WPAN $[3,6]$. In [3], the authors demonstrated the significant performance degradation of WLAN when the two networks coexist. Specifically, WLAN throughput is degraded more significantly as the ZigBee duty cycle or WLAN transmission rate increases. In addition, the UDP packet error rate increases and becomes very noticeable as the ZigBee duty cycle increases. This implies that retransmissions at the link layer could not completely shield the collisions and interference from the WPAN. Furthermore, the main reason behind the performance degradation of the WLAN is that the WPAN backoff slot is significantly longer than that of the WLAN ( 320 versus $20 \mu \mathrm{s}$, respectively), which reduces the effectiveness of the listen-before-send mechanisms of the two media access control (MAC) protocols.

Moreover, the work [7] confirmed that WLAN packet error rate does not significantly depend on the number and traffic of ZigBee nodes while ZigBee nodes experience much higher packet error rate when coexisting with the WLAN. Moreover, the ZigBee packet error rate increases significantly as the WLAN traffic arrival rate becomes larger. However, the ZigBee packet error rate can be improved considerably as we increase the ZigBee polling time or the ZigBee transmission power (or signal-to-interference ratio (SIR)). In [8], it was illustrated via experimental study that the ZigBee packet error rate increases with decreasing frequency offsets between communication bands of WLAN and WPAN. This is because the WLAN power spectral density is not uniform over the communication bandwidth. Moreover, it was shown again in this work that the WPAN can improve its packet error rate with higher transmission power. These existing works, therefore, demonstrate the mutual impacts between WLAN and WPAN, and the levels of the mutual impacts on packet error rate and throughput depend on different parameters including the relative distance between them, frequency offset, traffic arrival rate or duty cycle, communication rate, and transmission power.

There have been some efforts in developing more efficient coexistence solutions for WLAN and ZigBee networks. In [9, 10], the authors proposed to employ a ZigBee signaler to cooperate the transmissions of ZigBee and WLAN devices. The authors in [11, 12] proposed to exploit the silent periods during which WLAN nodes are idle for ZigBee nodes' communications. The work [13] developed the interference detection and channel switching method to enable ZigBee nodes to avoid WLAN interference. In [14], frequency hopping techniques were adopted to mitigate the coexistence interference. Nevertheless, none of these works provided any mathematical model for performance evaluation and enhancement of the coexisting networks.

Throughput analysis for the MAC protocol of either WLAN or ZigBee network under saturated and nonsaturated traffic conditions have been conducted separately in the literature. Bianchi was among the first who have developed mathematical models to evaluate the saturated performance of WLAN [15]. In [16], an alternative model based on the approximated p-persistent MAC protocol was devised for performance analysis and optimization of WLAN throughput. Performance study of the unsaturated system where WLAN nodes do not always have packets to transmit was conducted in [17]. The authors in [18] developed an analytical model for the IEEE 802.15.4 network based on the approximated non-persistent MAC under the unsaturated load condition. In [19], the authors proposed to employ renewal theory for analysis of the slotted non-persistent IEEE 802.15.4 MAC. The authors in [20] invented another analytical Markov chain (MC) model for the unsaturated slotted carrier sense multiple access with collision avoidance (CSMA/CA) of the IEEE 802.15.4 MAC considering the superframe structure. The MC model for throughput analysis of saturated and unsaturated IEEE 802.15.4 networks was developed in [21].

Mathematical throughput analysis of coexisting WLAN and ZigBee network is very underexplored in the literature. In [22], the authors performed mathematical analysis for the coexistence of WLAN and ZigBee network under some specific MAC and network settings. Specifically, this work assumed that the clear channel assessment (CCA) mode 2 is employed where each network node can only detect the transmission of other nodes of the same network (transmissions from WLAN nodes are transparent from or cannot be detected by ZigBee nodes and transmissions from ZigBee nodes are transparent from or cannot be detected by WLAN nodes). Moreover, the WLAN was assumed to broadcast data without using the standard CSMA/CA protocol and retransmitting erroneous packets. With these assumptions, the WLAN and WPAN are completely undetected from each other and the analysis is equivalent to studying the impacts of interference from one network to another.

We propose mathematical models to evaluate the coexistence performance of 802.15.4 ZigBee network and 802.11 WLAN. There is discrepancy in the transmission powers of WLAN and WPAN nodes; therefore, the impacts of mutual wireless interference on the throughput performance of WLAN and ZigBee network would depend on the coexistence situations and distances between the network nodes. In this paper, we consider two different scenarios, namely the symmetric and asymmetric ones. Specifically, the symmetric scenario represents the case where WLAN and ZigBee nodes can sense each 
other (i.e. all network nodes are sufficiently close to one another) whereas the asymmetric scenario describes the setting where ZigBee nodes can sense transmissions from WLAN nodes but WLAN nodes cannot sense the transmissions from ZigBee nodes. In addition, we study the general unsaturated traffic and develop the MC-based mathematical models for both scenarios where the interactions for a typical pair of WLAN and ZigBee nodes are studied considering detailed operations of both MAC protocols. Importantly, we assume that both networks employ the CCA mode 1, which detects a busy channel by comparing the measured signal strength or energy with a predetermined threshold. It is noted that the CCA mode 1 is more commonly used than the CCA mode 2 in practice [7].

Major challenges for developing such analytical models arise from the fact that the two MAC protocols of 802.15.4 WPAN and 802.11 WLAN operate in different time scales and they employ distinct backoff and CCA mechanisms. To overcome these obstacles, we propose to approximate the two MAC protocols by the corresponding p-persistent MAC protocols. Although this type of approximation has been adopted in analyzing the MAC throughput performance of individual networks (e.g. for 802.11 WLAN MAC in [16]), development of comprehensive mathematical models for performance analysis of the coexisting 802.15.4 ZigBee network and 802.11 WLAN is highly non-trivial. This indeed enables us to make important technical contributions toward understanding the complicated coexistence performance of the underlying MAC protocols. In addition, we also discuss how to employ the developed models for fair channel allocation between the two networks. Finally, we present numerical results to validate the proposed analytical model, demonstrate the impacts of different parameters on the throughput performance, and show the usefulness of the model in designing fair channel sharing for the considered heterogeneous environment.

The remaining of this paper is organized as follows. In Section 2, we describe both 802.15.4 and 802.11 MAC protocols and the considered coexistence scenarios. In Section 3, we present the MC-based analytical model for coexistence performance analysis in the symmetric scenario. In Section 4, we describe the throughput analysis for the asymmetric scenario. Application of the proposed framework for channel allocation is discussed in Section 5. We present the numerical results in Section 6 followed by conclusion in Section 7.

\section{MAC protocols and coexistence model}

In the following, we would like to briefly describe the MAC protocols of the 802.11 and 802.15.4 wireless networks. It is assumed that the reader is familiar with these protocols. For more information about the physical layer and MAC layer of the 802.11 and 802.15.4 with detailed MAC protocol description, the reader is referred to $[7,9,23-25]$.

\subsection{IEEE 802.15.4 MAC protocol}

We consider the IEEE 802.15.4 MAC protocol that operates in the beacon mode [23], where a PAN coordinator periodically sends the beacon for synchronization and each ZigBee node competes with others for transmission during the contention access period (CAP) by using the slotted CSMA/CA protocol. In this protocol, there are three important parameters, namely NB represents the number of attempts so far by the current packet, $C_{W}$ is the number of slots that need to be in the "clear" condition by sensing before a device that is allowed to access the channel, and $w$ denotes the number of backoff slots a device needs to wait before sensing the channel. $\mathrm{NB}$ is often initiated at 0 and upper-bounded by $\mathrm{NB}_{\max }=m_{z}$ while $C_{W}$ is set equal to 2 before each transmission attempt and reset to 2 each time channel is sensed busy.

At the beginning, each ZigBee node independently delays for a random number of slots $w$ chosen in the range from 0 to $2^{\mathrm{BE}_{\mathrm{NB}}}-1$ where $\mathrm{BE}_{0}=\operatorname{minBE}$ is the initial and minimum backoff exponent and then performs two clear CCAs. If the channel is sensed busy during either CCA, $C_{W}$ is reset to 2 , and NB increases by one. That means $\mathrm{BE}_{\mathrm{NB}+1}=\mathrm{BE}_{\mathrm{NB}}+1$, which is upper-bounded by maxBE. If $\mathrm{NB}$ is less than or equal to $\mathrm{NB}_{\max }$, the above backoff and CCA processes are repeated; otherwise, the transmission fails and the node can start this procedure again in the next frame. We denote the ZigBee backoff slot duration as $t_{\mathrm{bo}}$.

\subsection{IEEE 802.11 MAC protocol}

The IEEE 802.11 MAC protocol is the CSMA/CA-based contention-based access scheme [24]. In this protocol, a WLAN node needs to sense the channel while performing backoff, which is described in the following. If a channel is sensed idle during a Distributed Interframe Space (DIFS) interval, the node proceeds to perform backoff; otherwise, it defers its operation in the current busy period. When the channel is sensed idle for a DIFS interval, the node generates the random backoff delay uniformly chosen in the interval $[0, \mathrm{CW}-1]$ and starts counting down while listening to the medium.

Initially, the contention window size $\mathrm{CW}$ is set equal to the minimum value $\mathrm{CW}_{\min }$. The node decreases its backoff timer by one for every silent time slot period. Moreover, the backoff timer is suspended as long as the channel is sensed busy (there are transmissions from other nodes). The decrease of the backoff timer is resumed when a channel is sensed idle for a DIFS interval. When the backoff timer reaches zero, the node begins to transmit its packet. If the destination node receives a packet 
successfully, it waits for a Short Interframe Space (SIFS) interval, and then sends an ACK to the source node.

If the source node does not receive the ACK within an ACK timeout duration, it proceeds to retransmission. For each retransmission, the node doubles the contention window and enters the backoff delay process. If the contention window reaches the maximum contention window $C W_{\max }=2^{m_{w}} \mathrm{CW}_{\min }$, it remains unchanged until the transmission succeeds. We denote $\delta$ as the WLAN backoff slot duration. According to the 802.15.4 and 802.11 standards, the ZigBee backoff slot duration $t_{\text {bo }}$ is much larger than that of the WLAN slot duration $\delta$. This raises one major challenge in developing mathematical models to evaluate the coexistence performance besides the different operations of the two underlying MAC protocols.

\subsection{Coexistence model}

We consider the coexistence setting where the ZigBee network and WLAN operate on the same frequency channel and geographical area. We assume that WLAN and ZigBee users operate in the non-saturation mode where the users do not always have packets to transmit. The offered load due to each WLAN and ZigBee node is assumed to follow the Poisson distribution with average arrival rates $\lambda_{\mathrm{w}}$ and $\lambda_{\mathrm{z}}$ pkts/s, respectively. We further assume that packets arriving at the nodes are buffered awaiting transmissions. We consider the one-hop star topologies for WLAN and ZigBee network where the coordinators correspond to the access point (AP) in WLAN and PAN coordinator in ZigBee network (Fig. 1).

Since the transmission power of IEEE 802.11 WLAN node is much larger than that of IEEE 802.15.4 ZigBee nodes, the manner that one network impacts the other would depend on the relative distance between nodes in

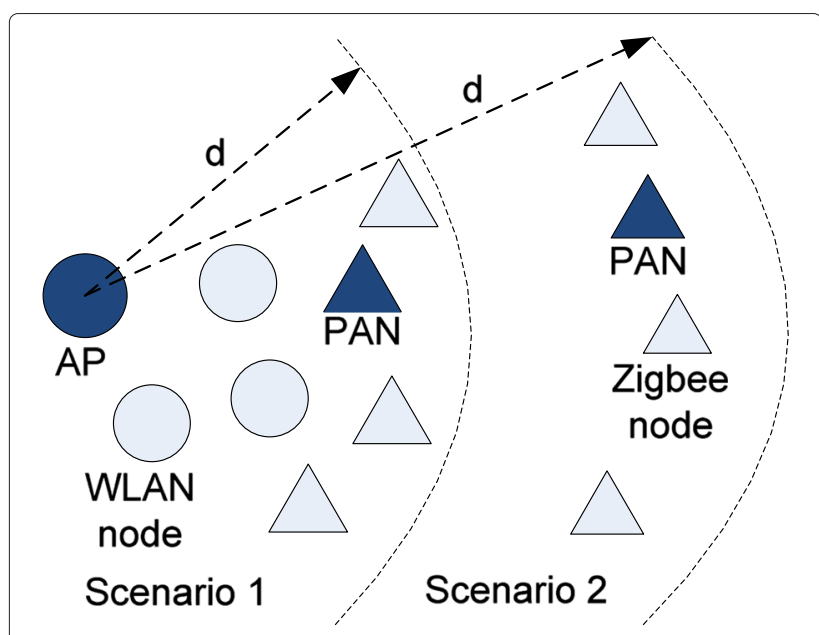

Fig. 1 Coexistence scenarios between WLAN and ZigBee network the two networks. To study the coexistence performance comprehensively, we consider two scenarios. In the first scenario (also called symmetric scenario), any node of either network can perfectly sense and detect the transmissions of any other nodes. This would be the case as the distance between WLAN nodes and ZigBee nodes are small (distance $d$ is small). For the second scenario (all called asymmetric scenario), ZigBee nodes can sense and detect the transmissions of WLAN nodes but the WLAN nodes cannot sense and detect the transmissions of ZigBee nodes. This would happen as the distance $d$ between WLAN nodes and ZigBee nodes are sufficiently large [2]. The considered coexistence scenarios are illustrated in Fig. 1.

\section{Performance analysis for symmetric scenario}

\subsection{Markov chain model}

In the considered setting, the nodes of either network are able to sense and detect transmissions from any other nodes. Therefore, we analyze the coexistence performance by defining a $\mathrm{MC}$ that captures the interactions for a typical pair of WLAN and ZigBee nodes. The behavior of the pair of the tagged (WLAN, ZigBee) nodes is characterized by the bi-dimensional discrete-time $\mathrm{MC}$ $\{a(t), b(t)\}$ where:

- $a(t)$ represents the states of the WLAN node including the backoff stages $W_{i}\left(i=1, \ldots, m_{w}\right)$ and idle state $I_{\mathrm{w}}$ at time $t$. From this definition, $W_{m_{w}}$ denotes the "maximum backoff stage" of the WLAN node.

- $b(t)$ describes the states of the ZigBee node comprising the idle state $I_{z}$, backoff stage $Z_{j}$ $\left(j=1, \ldots, m_{z}\right)$, the first CCA state $C_{1 j}$ and the second CCA state $C_{2, j}$ in backoff stage $j$, and transmission state $T_{x}$ of the ZigBee node at time $t$. Again, $Z_{m_{z}}$ represents the "maximum backoff stage" of the ZigBee node.

Note that most existing $\mathrm{MC}$ models for analyzing either IEEE 802.11 or IEEE 802.15.4 MAC protocol individually are based on two-dimensional MC (e.g. the models in [15] and [21]). Applying this approach for coexistence performance analysis of IEEE 802.11 and IEEE 802.15.4 networks would require to establish a four-dimensional $\mathrm{MC}$, which is too complex and non-tractable. By representing the states of each node in the considered (WLAN, ZigBee) pair by a single variable, we attain a much more manageable model. Modeling of the IEEE 802.15.4 MAC protocol using one-dimensional MC has been adopted in [18]; however, analyzing the coexistence performance of the two networks is far more challenging and still an open problem. Note also that the idle states $I_{\mathrm{w}}$ and $I_{\mathrm{z}}$ for WLAN and ZigBee nodes, respectively, are utilized to capture the cases where the buffers at these nodes are empty. 
To analyze the achievable throughput of each network in the considered heterogeneous environment, one has to characterize the transition probabilities of all possible transitions of the MC $\{a(t), b(t)\}$ based on which the steady-state probabilities can be calculated. Since the two MAC protocols of IEEE 802.11 and IEEE 802.15.4 networks operate in different time scales (i.e. $t_{\mathrm{bo}} \gg \delta$ ), we propose to analyze the $\{a(t), b(t)\}$ over the shorter time scale of WLAN and approximate the backoff mechanisms of both MAC protocols by the corresponding $p$-persistent MAC. In fact, this approximation has been confirmed to be very accurate for analyzing the WLAN MAC protocol [16]. We will validate the excellent accuracy of this approximation for coexistence performance analysis later in Section 6.

Specifically, in the approximated WLAN p-persistent MAC protocol, the random backoff time is drawn according to a geometric distribution with parameters $s_{i}$ in backoff stage $W_{i}$ where $s_{i}=2 /\left(\mathrm{CW}_{i}+1\right)$ and $\mathrm{CW}_{i}$ is the contention window in the backoff stage $W_{i}[16]$. That means if the channel is idle in a backoff slot, the WLAN node transmits and defers the transmission at backoff stage $W_{i}$ with probability $s_{i}$ and $\left(1-s_{i}\right)$, respectively. In contrast, if the channel is busy, no action is taken. The backoff stage increases when a transmission fails and the retry limit has not been reached. When a transmission succeeds, the WLAN node moves to the idle state $I_{\mathrm{W}}$ with probability $\left(1-q_{\mathrm{w}}\right)$ or to the first backoff stage $W_{1}$ with probability $q_{\mathrm{w}}$. We also assume that the WLAN transmission collides with probability $p$ or succeeds with probability $1-p$.

To resolve the heterogeneous time-scale issue, we consider the operations of the IEEE 802.15.4 MAC over the WLAN time slot unit. Toward this end, the length of the first and second CCAs, denoted as $L_{\mathrm{C}}$ and measured in WLAN backoff slot, is $L_{\mathrm{C}}=\frac{t_{\mathrm{bo}}}{\delta} L_{\mathrm{cca}}$ where $L_{\mathrm{cca}}$ is the length of CCA measured in the ZigBee backoff slot. We also approximate the IEEE 802.15.4 MAC protocol by the corresponding p-persistent MAC protocol. That means the random backoff time in the backoff stage $Z_{j}$ follows the geometric distribution with parameter $p_{j}$ where $p_{j}$ is calculated as [18]

$$
p_{j}=\frac{2}{2^{\mathrm{BE}_{j}} t_{\mathrm{bo}} / \delta+1} \text {. }
$$

Moreover, upon a successful transmission, the tagged ZigBee node changes to the idle state $I_{\mathrm{Z}}$ with probability $1-q_{\mathrm{z}}$ and to the first backoff state $Z_{1}$ with probability $q_{\mathrm{z}}$. At the backoff state $Z_{j}$, the ZigBee node remains in this backoff state with probability $\left(1-p_{j}\right)$ or enters the first CCA state $C_{1, j}$ with probability $p_{j}$. We assumed that the channel is sensed busy in the first CCA and the second CCA occurs with probabilities $\alpha$ and $\beta$, respectively. This is the case if there is at least one node rather than tagged pair of (WLAN, ZigBee) nodes that transmit their packets. In the following, we describe the transitions and the corresponding transition probabilities of the MC $\{a(t), b(t)\}$.

\subsection{Transition probabilities of Markov chain}

We can describe the state space $S$ of the $\mathrm{MC}\{a(t), b(t)\}$ as

$$
\begin{aligned}
& S=\left\{\text { all states } x:\left(I_{\mathrm{w}}, I_{\mathrm{Z}}\right),\left(I_{\mathrm{w}}, Z_{j}\right),\left(I_{\mathrm{w}}, C_{k, j}\right),\right. \\
& \left(I_{\mathrm{w}}, T_{x}\right),\left(W_{i}, I_{\mathrm{z}}\right),\left(W_{i}, Z_{j}\right),\left(W_{i}, C_{k, j}\right),\left(W_{i}, T_{x}\right), \\
& \left.i=1, \ldots, m_{w} ; j=1, \ldots, m_{z} ; k=1,2\right\} .
\end{aligned}
$$

To analyze this MC, we have to determine all possible transitions and the corresponding transition probabilities of this MC. To demonstrate the derivations, we present the transitions of the MC as the tagged WLAN node is in the idle state $I_{\mathrm{w}}$ considering all possible transitions between the states of the ZigBee node, which are shown in Fig. 2, in the following. The underlying transitions have the form $\left(I_{\mathrm{w}}, y\right) \rightarrow\left(I_{\mathrm{w}}, z\right)$ for the tagged (WLAN, ZigBee) nodes. Due to the space constraint, derivations for other transitions are given in the online technical report [26].

Here, the WLAN node remains in the idle state with probability $\left(1-q_{\mathrm{w}}\right)$ where there is no new packet arrival while the ZigBee node can transit to one of the possible states. Specifically, the transitions illustrated in Fig. 2 occur with the following transition probabilities ${ }^{1}$

$$
\begin{aligned}
& P\left\{I_{\mathrm{w}}, C_{1, j} \mid I_{\mathrm{w}}, Z_{j}\right\}=\left(1-q_{\mathrm{w}}\right) p_{j}, j \in\left[1, m_{z}\right] \\
& P\left\{I_{\mathrm{w}}, C_{2, j} \mid I_{\mathrm{w}}, C_{1, j}\right\}=\left(1-q_{\mathrm{w}}\right)(1-\alpha), j \in\left[1, m_{z}\right] \\
& P\left\{I_{\mathrm{w}}, T_{x} \mid I_{\mathrm{w}}, C_{2, j}\right\}=\left(1-q_{\mathrm{w}}\right)(1-\beta), j \in\left[1, m_{z}\right]
\end{aligned}
$$$$
P\left\{I_{\mathrm{w}}, C_{1, j} \mid I_{\mathrm{w}}, C_{k, j-1}\right\}=\left\{\begin{array}{l}
\left(1-q_{\mathrm{w}}\right) \alpha p_{j}, j \in\left(1, m_{z}\right], k=1 \\
\left(1-q_{\mathrm{w}}\right) \beta p_{j}, j \in\left(1, m_{z}\right], k=2
\end{array}\right.
$$$$
P\left\{I_{\mathrm{w}}, C_{1,1} \mid I_{\mathrm{w}}, C_{k, m_{z}}\right\}=\left\{\begin{array}{l}
\left(1-q_{\mathrm{w}}\right) \alpha q_{\mathrm{z}} p_{1}, k=1 \\
\left(1-q_{\mathrm{w}}\right) \beta q_{\mathrm{z}} p_{1}, k=2
\end{array}\right.
$$$$
P\left\{I_{\mathrm{w}}, Z_{j} \mid I_{\mathrm{w}}, C_{k, j-1}\right\}=\frac{\left(1-p_{j}\right)}{p_{j}} P\left\{I_{\mathrm{w}}, C_{1, j} \mid I_{\mathrm{w}}, C_{k, j-1}\right\}
$$

$$
P\left\{I_{\mathrm{w}}, Z_{1} \mid I_{\mathrm{w}}, C_{k, m_{z}}\right\}=\frac{\left(1-p_{1}\right)}{p_{1}} P\left\{I_{\mathrm{w}}, C_{1,1} \mid I_{\mathrm{w}}, C_{k, m_{z}}\right\}
$$

$$
P\left\{I_{\mathrm{w}}, I_{\mathrm{z}} \mid I_{\mathrm{w}}, C_{k, m_{z}}\right\}=\left\{\begin{array}{l}
\left(1-q_{\mathrm{w}}\right) \alpha\left(1-q_{\mathrm{z}}\right), k=1 \\
\left(1-q_{\mathrm{w}}\right) \beta\left(1-q_{\mathrm{z}}\right), k=1
\end{array}\right.
$$




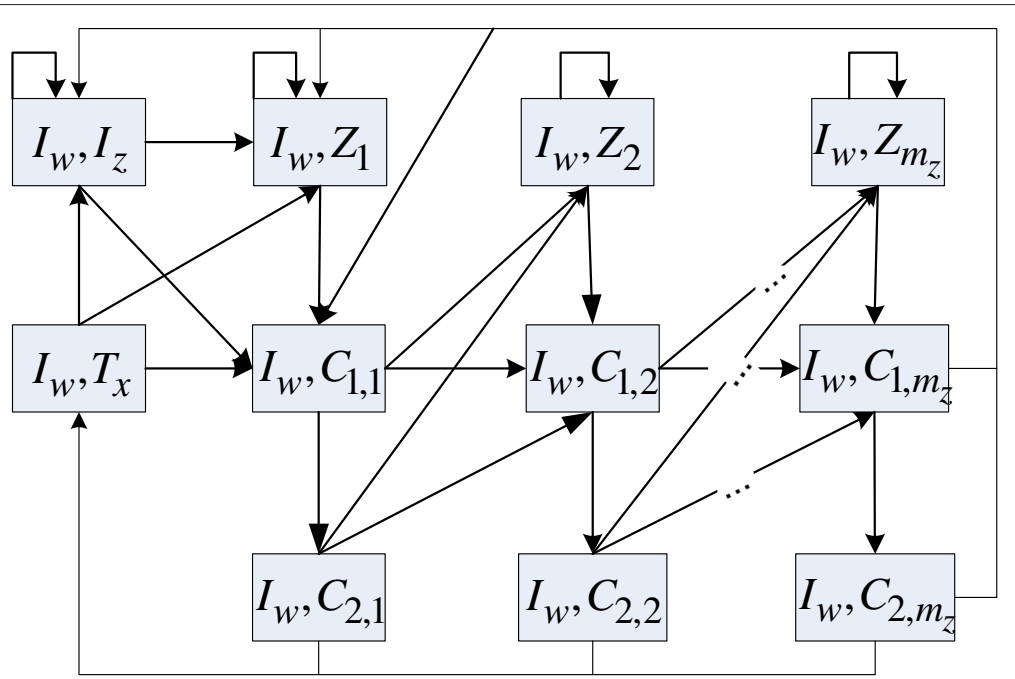

Fig. 2 Transitions of the form $\left(I_{w}, y\right) \rightarrow\left(I_{w}, z\right)$ of (WLAN, ZigBee) nodes

$P\left\{I_{\mathrm{w}}, Z_{1} \mid I_{\mathrm{w}}, T_{x}\right\}=P\left\{I_{\mathrm{w}}, Z_{1} \mid I_{\mathrm{w}}, I_{\mathrm{z}}\right\}=\left(1-q_{\mathrm{w}}\right) q_{\mathrm{z}}\left(1-p_{1}\right)$

$$
P\left\{I_{\mathrm{w}}, C_{1,1} \mid I_{\mathrm{w}}, T_{x}\right\}=P\left\{I_{\mathrm{w}}, C_{1,1} \mid I_{\mathrm{w}}, I_{\mathrm{z}}\right\}=\left(1-q_{\mathrm{w}}\right) q_{\mathrm{z}} p_{1}
$$

$$
P\left\{I_{\mathrm{w}}, I_{\mathrm{z}} \mid I_{\mathrm{w}}, T_{x}\right\}=P\left\{I_{\mathrm{w}}, I_{\mathrm{z}} \mid I_{\mathrm{w}}, I_{\mathrm{z}}\right\}=\left(1-q_{\mathrm{w}}\right)\left(1-q_{\mathrm{z}}\right) .
$$

Equation (3) captures the case where the tagged ZigBee node finishes the backoff in the backoff stages $Z_{j}$ and proceeds to the first CCA state $C_{1, j}$. Equation (4) represents the case the channel is sensed idle during the first CCA with probability $(1-\alpha)$ after which the ZigBee node enters the second CCA. In (5), we consider the transition of the ZigBee node from the idle channel state during the second CCA to the transmitting state. Equations (6) and (7) describe the scenarios where the ZigBee node experiences the busy channel in the first and second CCAs, respectively. In these cases, the ZigBee node enters the first CCA of the next backoff stage in Eq. (6) or the first CCA of the first backoff stage when it is at the maximum backoff state and its buffer is backlogged in Eq. (7).

Similarly, Eqs. (8) and (9) capture the transitions where the ZigBee node experiences all busy CCAs, and it enters the next backoff stage. Equation (10) corresponds to transitions where the ZigBee node experiences the busy channel for all CCAs at the maximum backoff stage and its buffer is empty; consequently, it enters the idle state. For transitions whose probabilities are given in (11) and (12), the ZigBee node finishes the transmission, its buffer is backlogged, and it evolves to either the first backoff stage or the first CCA of the first backoff stage, respectively. Equation (13) accounts for the case where the ZigBee node's buffer is empty after its transmission; therefore, it enters the idle state.

\subsection{Throughput analysis}

Recall that we employ either $x$ or explicit description $\{a(t), b(t)\}$ to specify a particular state of the MC. Let $\pi(x)$ denote the steady probability of state $x$, which is in the state space described in (2). Suppose we arrange all the states $x$ such that their indices range from 0 to $v_{\max }$. In order to calculate the steady-state distribution of the $\mathrm{MC}$, we can write all transition probabilities into the corresponding matrix $\mathbf{P}$ whose elements $P\left\{x^{\prime} \mid x\right\}$ denote the transition probability from states $x$ to states $x^{\prime}$. Then, the steady-state probabilities can be determined by solving the following equations:

$$
\pi \mathbf{P}=\pi ; \quad \sum_{x=0}^{v_{\max }} \pi(x)=1 .
$$

where $\pi$ denotes the steady-state probability vector, which is defined as $\pi=\left[\pi(0), \ldots, \pi\left(v_{\max }\right)\right]$. These steady-state probabilities $\pi$ are the functions of $p, q_{\mathrm{w}}, q_{\mathrm{z}}, \alpha$, and $\beta$.

The probability that one node (either WLAN or ZigBee node) attempts to transmit in a generic backoff slot $\tau$ is equal to the total probability of the following events: the ZigBee node is in one of the backoff states, it senses the idle channel in the second CCA, and the WLAN node starts transmission at one of the backoff stages. The dwell time of the states in which the ZigBee node is in the transmission states (i.e. states $\left(I_{\mathrm{w}}, T_{x}\right)$ and $\left.\left(W_{i}, T_{x}\right)\right)$ and the sensing states (i.e. states $\left(I_{\mathrm{w}}, C_{k, j}\right)$ and $\left.\left(W_{i}, C_{k, j}\right)\right)$ are $T_{z}$ and $L_{\mathrm{C}}$, respectively where $i=1, \ldots, m_{w}, j=1, \ldots, m_{z}$ and $k=1,2$; and the dwell time of all other states are 1 . Hence, the transmission probability $\tau$ can be calculated as in (15) where $\Psi$ is given in (16). 


$$
\begin{aligned}
\tau= & \frac{1}{\psi}\left(\sum_{i=1}^{m_{w}} s_{i} \sum_{j=1}^{m_{z}}\left[\pi\left(W_{i}, Z_{j}\right)+L_{C} \pi\left(W_{i}, C_{1, j}\right)\right]\right. \\
& +L_{C} \sum_{j=1}^{m_{z}} \sum_{i=1}^{m_{w}}\left(1-\beta+s_{i}\right) \pi\left(W_{i}, C_{2, j}\right) \\
& \left.+L_{\mathrm{C}}(1-\beta) \sum_{j=1}^{m_{z}} \pi\left(I_{\mathrm{w}}, C_{2, j}\right)+\sum_{i=1}^{m_{w}} s_{i} \pi\left(W_{i}, I_{\mathrm{z}}\right)\right)
\end{aligned}
$$

$$
\begin{aligned}
\psi= & \sum_{j=1}^{m_{z}}\left[\sum_{i=1}^{m_{w}} \pi\left(W_{i}, Z_{j}\right)+\sum_{k=1}^{2} \pi\left(W_{i}, C_{k, j}\right) L_{\mathrm{C}}\right] \\
& +\sum_{i=1}^{m_{w}}\left[T_{z} \pi\left(W_{i}, T_{x}\right)+\pi\left(W_{i}, I_{\mathrm{z}}\right)\right]+T_{z} \pi\left(I_{\mathrm{w}}, T_{x}\right)+\pi\left(I_{\mathrm{w}}, I_{\mathrm{z}}\right) \\
& +\sum_{j=1}^{m_{z}}\left[\pi\left(I_{\mathrm{w}}, Z_{j}\right)+\sum_{k=1}^{2} \pi\left(I_{\mathrm{w}}, C_{k, j}\right) L_{\mathrm{C}}\right]
\end{aligned}
$$

Similar to the derivation of $\tau$, the probability that a WLAN node attempts to transmit in a generic slot $\tau_{w}$, the probability that a ZigBee node starts sensing in a generic slot $\phi$, and the probability that the ZigBee node attempts to transmit in a generic slot $\tau_{z}$ are given respectively as

$$
\begin{array}{r}
\tau_{W}=\frac{1}{\psi}\left(\sum _ { i = 1 } ^ { m _ { w } } s _ { i } \sum _ { j = 1 } ^ { m _ { z } } \left[\pi\left(W_{i}, Z_{j}\right)+\right.\right. \\
\left.\sum_{k=1}^{2} \pi\left(W_{i}, C_{k, j}\right) L_{\mathrm{C}}\right] \\
\left.+\sum_{i=1}^{m_{w}} s_{i} \pi\left(W_{i}, I_{\mathrm{z}}\right)\right)
\end{array}
$$

$$
\begin{array}{r}
\phi=\frac{1}{\psi}\left[\sum_{i=1}^{m_{w}} \sum_{j=1}^{m_{z}} p_{j} \pi\left(W_{i}, Z_{j}\right)+\sum_{j=1}^{m_{z}} p_{j} \pi\left(I_{\mathrm{w}}, Z_{j}\right)\right] \\
\tau_{z}=\frac{(1-\beta) L_{\mathrm{C}}}{\psi}\left(\sum_{i=1}^{m_{w}}\left(1-s_{i}\right) \sum_{j=1}^{m_{z}} \pi\left(W_{i}, C_{2, j}\right)\right. \\
\left.+\sum_{j=1}^{m_{z}} \pi\left(I_{\mathrm{w}}, C_{2, j}\right)\right) .
\end{array}
$$

The collision probability $p$ is calculated as $p=1-(1-$ $\tau)^{(n-1)}$ [15] for $n=n_{\mathrm{w}}+n_{\mathrm{z}}$ where $n_{\mathrm{w}}$ and $n_{\mathrm{z}}$ denote the number of WLAN and ZigBeez nodes, respectively. The expected length of a generic WLAN slot $E\left[S_{t_{w}}\right]$ can be calculated as ${ }^{2}$

$$
\begin{aligned}
E\left[S_{t s_{w}}\right]= & \left(1-P_{\mathrm{t}}\right) \delta+P_{\mathrm{tw}}\left(1-P_{\mathrm{s}}\right)\left(1-P_{\mathrm{tz}}\right) T_{c w} \\
& +P_{\mathrm{tw}} P_{\mathrm{tz}} T_{z}+P_{\mathrm{tw}} P_{\mathrm{s}} T_{s}+P_{\mathrm{tz}}\left(1-P_{\mathrm{s}}\right) T_{z}+P_{\mathrm{tz}} P_{\mathrm{s}} T_{z}
\end{aligned}
$$

where $P_{\mathrm{t}}$ is the probability that there is at least one transmission (either WLAN transmission or ZigBee transmission); $P_{\mathrm{tw}}$ and $P_{\mathrm{tz}}$ denote the probabilities that there is at least one WLAN transmission and one ZigBee transmission in the considered slot, respectively; and $P_{\mathrm{s}}$ represents the conditional probability that there is exactly one node transmitting in a given slot. We can compute these probabilities as follows:

$$
\begin{aligned}
P_{\mathrm{t}} & =1-(1-\tau)^{n} ; P_{\mathrm{tw}}=1-\left(1-\tau_{w}\right)^{n_{\mathrm{w}}} \\
P_{t z} & =1-\left(1-\tau_{z}\right)^{n_{\mathrm{z}}} ; P_{\mathrm{s}}=n \tau(1-\tau)^{n-1} / P_{\mathrm{t}} .
\end{aligned}
$$

The probabilities $q_{\mathrm{w}}$ and $q_{\mathrm{z}}$ that there is at least one packet to be transmitted from the MAC buffer of a WLAN or ZigBee node can be estimated as [17]

$$
q_{\mathrm{w}}=1-e^{-\lambda_{\mathrm{w}} E\left[S_{t_{w}}\right]} ; q_{\mathrm{z}}=1-e^{-\lambda_{\mathrm{z}} E\left[S_{t_{w}}\right]}
$$

where $\lambda_{w}$ and $\lambda_{z}$ are the packet arrival rates of WLAN and ZigBee nodes, respectively. Therefore, $q_{\mathrm{w}}$ and $q_{\mathrm{z}}$ are the functions of $\tau, \lambda_{\mathrm{w}}$ and $\lambda_{\mathrm{z}}$.

We now derive the probabilities $\alpha$ and $\beta$ that the channel is busy in the first and second CCAs, respectively. Let $P_{i \mid i}$ denote the probability that the channel progresses to the idle state in the next slot given that it is idle in the current slot where these slots are the ZigBee backoff slots. The probability that the channel is idle in a general ZigBee slot can be computed as

$$
P_{i}=P_{i \mid i} P_{i}+P_{i \mid b}\left(1-P_{i}\right)
$$

where $P_{i \mid b}$ denotes the probability that the channel becomes idle given that it is busy at the current slot. Since a busy duration lasts for $L=P_{\mathrm{tw}}\left(1-P_{\mathrm{s}}\right)\left(1-P_{\mathrm{tz}}\right) T_{\mathrm{cw}}+$ $P_{\mathrm{tw}} P_{\mathrm{s}} T_{s}+P_{\mathrm{tw}} P_{\mathrm{tz}} T_{z}+P_{\mathrm{tz}} T_{z}$ on average, a busy slot will end with probability $\frac{1}{L}$ during a random slot [19]. There is always an idle slot after the end of a busy slot for ZigBee node. Therefore, an idle slot follows the end of the busy slot occurs if none of WLAN nodes transmits during one ZigBee backoff slot, which yields

$$
P_{i \mid b}=\frac{1}{L}\left(1-P_{\mathrm{tw}}\right)^{L_{\mathrm{C}}} \text {. }
$$

Let $P_{b \mid i}$ denote the probability that the channel is busy in current slot $k$ given that it is idle in the previous slot $k-1$ measured in the ZigBee backoff slot. This can occur when the channel is idle in slot $k-2$ and at least one ZigBee node performs sensing in that time slot or there is at least one WLAN node transmitting at any WLAN slots in slot $k$ given none of the node transmits during slot $k-1$. Hence,

$$
P_{b \mid i}=\left(1-(1-\phi)^{n_{\mathrm{z}}}\right) P_{i \mid i}+\left(1-P_{\mathrm{t}}\right)^{L_{\mathrm{C}}} P_{\mathrm{tw}} L_{\mathrm{C}} .
$$


In addition, we have

$$
P_{i \mid i}=1-P_{b \mid i}
$$

From (26) and (27), we have

$$
P_{i \mid i}=\frac{1-\left(1-P_{\mathrm{t}}\right)^{L_{\mathrm{C}}} P_{\mathrm{tw}} L_{\mathrm{C}}}{1+\left(1-(1-\phi)^{n_{\mathrm{z}}}\right)} .
$$

Substituting the results in (25) and (28) into (24), we have $P_{i}$ is equal to $1-\alpha$.

Finally, the probability that the channel is found to be idle in the second CCA state given the channel is idle in the first CCA is equal to $1-\beta$, which is indeed $P_{i \mid i}$. Therefore, $\alpha$ and $\beta$ are the functions of $\tau, \tau_{w}, \tau_{z}$ and $\phi$ through $P_{i}$ and $P_{i \mid i}$. Hence, we have a system of equations based on which the steady-state probabilities, and probabilities $p, q_{\mathrm{w}}, q_{\mathrm{z}}, \alpha, \beta$ can be found by using the numerical method. The throughput achieved by each ZigBee and WLAN nodes can be expressed as

$$
\begin{aligned}
& S_{\mathrm{z}}=\frac{T_{z} n_{\mathrm{z}} \tau_{z}(1-\tau)^{n-1}}{E\left[S_{t_{w}}\right]} \\
& S_{\mathrm{w}}=\frac{T_{s} n_{\mathrm{w}} \tau_{w}(1-\tau)^{n-1}}{E\left[S_{t_{w}}\right]}
\end{aligned}
$$

which completes the throughput analysis.

\section{Performance analysis for asymmetric scenario}

In this scenario, the WLAN nodes cannot sense and detect the transmissions of the ZigBee nodes. This means that a particular WLAN node senses the idle channel whenever there is no other WLAN transmissions and it will transmit its packet when its backoff counter reaches zero.

\subsection{Analytical model}

Since WLAN nodes cannot sense ongoing transmissions from ZigBee nodes, WLAN nodes can start transmissions during ZigBee node's transmission. We assume that a WLAN transmission collides with other WLAN transmissions with probability $P_{\mathrm{cw}}$ while WLAN collisions due to ZigBee transmissions occur with probability $P_{\text {cz }}$. Hence, the WLAN transmission is successful if there is no collision with other WLAN and ZigBee transmissions, which happens with probability $\left(1-P_{\mathrm{CW}}\right)\left(1-P_{\mathrm{CZ}}\right)$. This yields the collision probability of a WLAN node

$$
P_{c}=P_{\mathrm{cW}}+P_{\mathrm{cz}}-P_{\mathrm{cW}} P_{\mathrm{cz}} \text {. }
$$

It can be observed that the WLAN performance can be analyzed similarly to the non-coexisting scenario with the ZigBee network with the new collision probability $P_{c}$. Therefore, we perform the analysis for WLAN and ZigBee network separately in the following.

\subsubsection{WLAN analytical model}

The analysis of WLAN in the non-coexisting scenario and unsaturated traffic has been studied using the two dimensional MC model in [17], whose important derivations are given here for completeness. The transmission probability of a WLAN node $\tau_{w}$ is given in Eq. (32) where $P_{\mathrm{cw}}=1-\left(1-\tau_{w}\right)^{n_{\mathrm{w}}-1}$.

$\tau_{w}=\frac{2\left(1-2 P_{c}\right) q_{\mathrm{w}}}{q_{\mathrm{w}}\left[(W+1)\left(1-2 P_{c}\right)+W P_{c}\left(1-\left(2 P_{c}\right)^{m_{w}}\right)\right]+2\left(1-q_{\mathrm{w}}\right)\left(1-P_{c}\right)\left(1-2 P_{c}\right)}$.

The probability that there is at least one WLAN transmission in the considered generic time slot is given as

$$
P_{\mathrm{tw}}=1-\left(1-\tau_{w}\right)^{n_{\mathrm{w}}}
$$

Let $P_{S}$ be the conditional probability that a WLAN transmission is successful, which is given by

$$
P_{s}=\frac{n_{\mathrm{w}} \tau_{w}\left(1-\tau_{w}\right)^{n_{\mathrm{w}}-1}}{P_{\mathrm{tw}}} .
$$

Let $E\left[S_{\mathrm{tw}}\right]$ denote the expected length of a generic WLAN slot, which does not depend on the existence of the ZigBee network. Here, this value is calculated considering the empty WLAN time slot, WLAN collision duration, and WLAN successful transmission duration. Hence, $E\left[S_{\mathrm{tw}}\right]$ can be computed as

$$
\begin{aligned}
E\left[S_{\mathrm{tw}}\right] & =\left(1-P_{\mathrm{tw}}\right) \delta+P_{\mathrm{tw}} P_{s}\left(1-P_{\mathrm{cz}}\right) T_{s} \\
& +P_{\mathrm{tw}}\left(1-P_{s}\right) T_{c w}+P_{\mathrm{tw}} P_{s} P_{\mathrm{cz}} T_{c w} .
\end{aligned}
$$

The probability $q_{\mathrm{w}}$ is given as

$$
q_{\mathrm{w}}=1-e^{-\lambda_{\mathrm{w}} E\left[S_{\mathrm{tw}}\right]} .
$$

\subsubsection{ZigBee analytical model}

We define the following one-dimensional MC for a tagged ZigBee node $Z(t)=\{b(t)\}$ where $b(t)$ represents the states of a ZigBee node. Specifically, we can describe the state space $Z$ of this $M C$ as

$$
Z=\left\{\text { all state } y: I_{\mathrm{z}}, Z_{j}, C_{k, j}, T_{x}, j=1, \ldots, m_{z} ; k=1,2\right\} .
$$

Similar to Section 3, we approximate the ZigBee backoff counter by a geometric random variable with parameter $p_{j}$ during the backoff stage $Z_{j}$ as given in (1). Since each ZigBee node independently determines its backoff value and automatically decreases the backoff counter, the channel sensing procedure of each ZigBee node at two CCAs depends on the WLAN transmissions. Suppose that the channel is sensed busy at the first and second CCA by a tagged ZigBee node with probabilities $\alpha$ and $\beta$, respectively. Denote $P\{y \mid y\}$ as the transition probability from state $y$ to state $y$. Transitions among different states of a 
tagged ZigBee node are shown in Fig. 3. The probabilities of all possible transitions are listed as follows:

$$
\begin{aligned}
P\left\{I_{\mathrm{z}} \mid I_{\mathrm{z}}\right\} & =P\left\{I_{\mathrm{z}} \mid T_{x}\right\}=1-q_{\mathrm{z}} \\
P\left\{Z_{1} \mid I_{\mathrm{z}}\right\} & =q_{\mathrm{z}}\left(1-p_{1}\right) \\
P\left\{Z_{1} \mid I_{\mathrm{z}}\right\} & =q_{\mathrm{z}} p_{1} \\
P\left\{Z_{j} \mid C_{k, j-1}\right\} & =\left\{\begin{array}{l}
\alpha\left(1-p_{j}\right) ; k=1, j=2, \ldots, m_{z} \\
\beta\left(1-p_{j}\right) ; k=2, j=2, \ldots, m_{z}
\end{array}\right. \\
P\left\{C_{1, j} \mid C_{k, j-1}\right\} & =\left\{\begin{array}{l}
\alpha p_{j} ; k=1, j=2, \ldots, m_{z} \\
\beta p_{j} ; k=2, j=2, \ldots, m_{z}
\end{array}\right. \\
P\left\{I_{\mathrm{z}} \mid C_{k, m_{z}}\right\} & =\left\{\begin{array}{l}
\alpha\left(1-q_{\mathrm{z}}\right) ; k=1 \\
\beta\left(1-q_{\mathrm{z}}\right) ; k=2
\end{array}\right. \\
P\left\{Z_{1} \mid C_{k, m_{z}}\right\} & =q_{\mathrm{z}} P\left\{B Z_{j} \mid C_{k, j-1}\right\} \\
P\left\{C_{1,1} \mid C_{k, m_{z}}\right\} & =q_{\mathrm{z}} P\left\{C_{1, j} \mid C_{k, j-1}\right\} \\
P\left\{C_{1, j} \mid Z_{j}\right\} & =p_{j} ; j=1, \ldots, m_{z} \\
P\left\{C_{2, j} \mid C_{1, j}\right\} & =(1-\alpha) ; j=1, \ldots, m_{z} \\
P\left\{T_{x} \mid C_{2, j}\right\} & =(1-\beta) ; j=1, \ldots, m_{z} .
\end{aligned}
$$

Let $\pi(y)$ denote the steady probability of state $y$, which belongs to the state space $Z$. Suppose we arrange all states whose indices range from 0 to $u_{\max }$. Let $\mathbf{P}$ denote the one-step transition probability matrix and $\pi$ denote the steady-state probability vector, which is defined as $\pi=$ $\left[\pi(0), \ldots, \pi\left(u_{\max }\right)\right]$. Here, the elements of matrix $\mathbf{P}$ are $p_{y, y}=P\{y \mid y\}$, which are computed in (38). We can calculate the steady-state probabilities of all the states vy solving the following set of equations

$$
\boldsymbol{\pi} \mathbf{P}=\boldsymbol{\pi} ; \quad \sum_{y=0}^{u_{\max }} \pi(y)=1 .
$$

Note that these steady-state probabilities are functions of $\alpha, \beta$, and $q_{\mathrm{z}}$.

\subsection{Throughput analysis}

The probabilities that a ZigBee node starts sensing and attempts to transmit in a generic slot are given, respectively as

$$
\begin{aligned}
\phi & =\frac{1}{\psi} \sum_{j=1}^{m_{z}} p_{j} \pi\left(Z_{j}\right) \\
\tau_{z} & =\frac{(1-\beta)}{\psi} \sum_{j=1}^{m_{z}} \pi\left(C_{2, j}\right)
\end{aligned}
$$

where

$$
\psi=\pi\left(I_{\mathrm{z}}\right)+T_{z} \pi\left(T_{x}\right)+\sum_{j=1}^{m_{z}} \pi\left(Z_{j}\right)+L_{C} \sum_{j=1}^{m_{z}} \sum_{k=1}^{2} \pi\left(C_{k, j}\right) .
$$

Let $P_{\mathrm{tz}}$ be the probability that there is at least one ZigBee transmissions in the considered slot, which is given by

$$
P_{\mathrm{tz}}=1-\left(1-\tau_{z}\right)^{n_{\mathrm{z}}} .
$$

Now, we determine the WLAN collision probability $P_{\mathrm{cz}}$ due to ZigBee transmissions. This collision event indeed occurs as a WLAN transmission encounters at least one ZigBee transmission. Since the WLAN and ZigBee are quite separated geographically in the asymmetric scenario, we assume that the WLAN transmission can be corrupted by the ZigBee transmissions with probability $\gamma$ where $\gamma \leq 1$. In addition, the WLAN collision probability $P_{\text {cz }}$ can be expressed as

$$
P_{\mathrm{cz}}=\gamma P_{\mathrm{tz}}
$$

Recall that the ZigBee node can still sense successfully the WLAN transmissions in the considered asymmetric scenario. Therefore, the expected length of a generic ZigBee slot $E\left[S_{\mathrm{tz}}\right]$ can be obtained by considering the

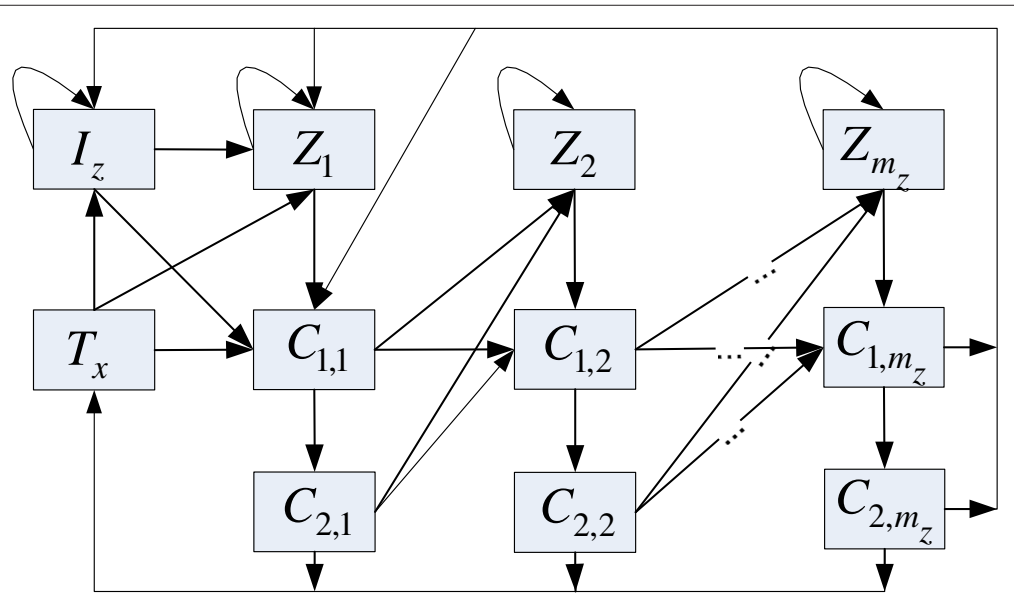

Fig. 3 Markov chain for tagged IEEE 802.15.4 WPAN node 
durations and the corresponding probabilities of the idle slot $\left(t_{\mathrm{bo}}\right)$ and the busy slot $\left(T_{z}, T_{s}\right.$ or $\left.T_{c w}\right)$ as follows:

$$
\begin{aligned}
E\left[S_{\mathrm{tz}}\right]= & \left(1-P_{\mathrm{t}}\right) t_{\mathrm{bo}}+P_{\mathrm{tz}} T_{z}+P_{\mathrm{tw}} P_{s}\left(1-P_{\mathrm{cz}}\right) T_{s} \\
& +P_{\mathrm{tw}}\left(1-P_{s}\right) T_{c w}+P_{\mathrm{tw}} P_{s} P_{\mathrm{cz}} T_{c w}
\end{aligned}
$$

where $P_{\mathrm{t}}$ represents the probability that there is at least one transmission (either WLAN or ZigBee transmission) in the considered time slot

$$
P_{\mathrm{t}}=1-\left(1-\tau_{w}\right)^{n_{\mathrm{w}}}\left(1-\tau_{z}\right)^{n_{\mathrm{z}}} .
$$

Then, the probability $q_{\mathrm{z}}$ is obtained as

$$
q_{\mathrm{z}}=1-e^{-\lambda_{\mathrm{z}} E\left[S_{\mathrm{tz}}\right]} \text {. }
$$

To compute the $\tau_{z}$ and $\phi$, we need to know $\alpha$ and $\beta$, which can be derived in a similar manner with that in Section 3 . In particular, the probability $P_{i \mid b}$ is given by

$$
P_{i \mid b}=\frac{1}{L}\left(1-P_{\mathrm{tw}}\right)^{L_{\mathrm{C}}}
$$

where

$$
\begin{aligned}
L= & P_{\mathrm{tz}} T_{z}+P_{\mathrm{tw}} P_{s}\left(1-P_{\mathrm{cz}}\right) T_{s}+P_{\mathrm{tw}}\left(1-P_{s}\right) T_{c w} \\
& +P_{\mathrm{tw}} P_{s} P_{\mathrm{cz}} T_{c w} .
\end{aligned}
$$

The probability $P_{b \mid i}$ is computed similarly to (26)

$$
P_{b \mid i}=\left(1-(1-\phi)^{n_{\mathrm{z}}}\right) P_{i \mid i}+\left(1-P_{\mathrm{t}}\right)^{L_{\mathrm{C}}} P_{\mathrm{tw}} L_{\mathrm{C}} .
$$

Using the results in (50) and (27), we can obtain $P_{i \mid i}$. Then, substituting $P_{i \mid i}$ and the results in (48) into (24), we obtain the $P_{i}$. Hence, the probability that a channel is sensed busy in the first CAA $\alpha$ and the second CCA $\beta$ can be written as $\alpha=P_{i}$ and $\beta=P_{i \mid i}$. Then, all the probabilities can be solved by using the numerical method. Finally, the throughput achieved by the ZigBee and WLAN node can be expressed as

$$
\begin{aligned}
S_{\mathrm{z}} & =\frac{T_{z} n_{\mathrm{z}} \tau_{z}\left(1-\tau_{w}\right)^{n_{\mathrm{w}}}\left(1-\tau_{z}\right)^{n_{\mathrm{z}}-1}}{E\left[S_{\mathrm{tz}}\right]} \\
S_{\mathrm{w}} & =\frac{T_{s} n_{\mathrm{w}} \tau_{w}\left(1-\tau_{w}\right)^{n_{\mathrm{w}}-1}\left(1-P_{\mathrm{cz}}\right)}{E\left[S_{\mathrm{tw}}\right]} .
\end{aligned}
$$

Therefore, we have completed the throughput analysis.

\section{Fair channel allocation}

We describe how to distribute the ZigBee nodes over overlapped and not overlapped channels, which are shared and not shared with WLAN nodes, respectively, to achieve fair throughput sharing among ZigBee nodes by using the proposed analytical models. Let $\Omega_{\text {over }}$ and $\Omega_{\text {non }}$ represent the sets of overlapping and non-overlapping channels of ZigBee network and WLAN, respectively.

To determine the channel allocation for ZigBee nodes, let $S_{\text {over }}^{k}\left(\lambda_{\mathrm{w}}, n_{\mathrm{w}}, \lambda_{\mathrm{z}}, n_{\mathrm{k}}\right)$ be the throughput achieved by a ZigBee node on the overlapping channel $f_{k}\left(f_{k} \in \Omega_{\text {over }}\right)$ where $\lambda_{\mathrm{w}}$ is the average arrival rate of WLAN, $n_{\mathrm{w}}$ is the number of WLAN nodes occupying channel $f_{k}, \lambda_{z}$ is the average arrival rate of ZigBee nodes, and $n_{\mathrm{k}}$ is number of ZigBee nodes assigned channel $f_{k}$. Also, let $S_{\text {non }}^{k}\left(\lambda_{z}, n_{\mathrm{k}}\right)$ be the throughput achieved by a ZigBee node on the nonoverlapping channel $f_{k}\left(f_{k} \in \Omega_{\text {non }}\right)$. These throughput measures can be calculated by using the analytical models presented in the previous Sections 3 and 4 depending on the considered coexistence scenario, which are assumed to be stored in a look-up table.

We are interested in assigning the ZigBee nodes to all channels so that the throughput achieved by each ZigBee node is as even as possible (the perfect scenario is where the throughput of all ZigBee nodes is the same). Here, we assume that the traffic load of WLAN (represented by its average packet arrival rate $\lambda_{\mathrm{W}}$ ) and the number of WLAN nodes $n_{\mathrm{w}}$ on each overlapping channel are the same for simplicity even though this assumption can be relaxed. ${ }^{3}$ Suppose there are $n_{\mathrm{z}}$ ZigBee nodes. Let $n_{1}$ and $n_{2}$ be the number of ZigBee nodes assigned to each overlapping and non-overlapping channel, respectively. Then, we obtain the assignment solution by solving the following optimization problem

$$
\begin{aligned}
\min & \Delta S\left(n_{1}, n_{2}\right)=\left|S_{\text {non }}^{l}\left(\lambda_{\mathrm{z}}, n_{2}\right)-S_{\text {over }}^{k}\left(\lambda_{\mathrm{w}}, n_{\mathrm{w}}, \lambda_{\mathrm{z}}, n_{1}\right)\right| \\
& \forall f_{l} \in \Omega_{\text {non }}, f_{k} \in \Omega_{\text {over }} \\
& \text { s.t. } n_{1}\left|\Omega_{\text {over }}\right|+n_{2}\left|\Omega_{\text {non }}\right|=n_{\mathrm{z}} .
\end{aligned}
$$

Since $n_{1}$ and $n_{2}$ can only take integer values, the constraint in problem (53) can be written as $n_{2}=$ $\left\lceil\frac{n_{z}-n_{1}\left|\Omega_{\text {over }}\right|}{\left|\Omega_{\text {non }}\right|}\right\rceil$. Since there are only a finite number of possible choices for $\left(n_{1}, n_{2}\right)$, we can easily search for the one that achieves the minimal objective.

\section{Numerical results}

We evaluate the ZigBee throughput performance when the ZigBee network coexists with the WLAN network on the same frequency channel. We assume that the packet arrivals of each WLAN and ZigBee node follow the Poisson distribution with mean value of $\lambda_{\mathrm{w}}$ and $\lambda_{\mathrm{z}} \mathrm{pkt} / \mathrm{s}$, respectively. In the asymmetric scenario, we assume the probability $\gamma$ is set to 0.5 . To obtain the numerical results presented in this section, we choose the MAC parameters for IEEE 802.11 and IEEE 802.15.4 network as detailed in Table 1.

For the considered coexistence model, we assume there are $n_{\mathrm{W}}$ WLAN nodes and $n_{\mathrm{z}}$ ZigBee nodes, respectively. Also, we consider $m_{w}=5$ for WLAN and $m_{z}=5$ for the Zibgee network. We also evaluate the throughput of ZigBee network assuming that the values of CCA, minBE, and $\operatorname{maxBE}$ are set equal to 2,3 , and 5 , respectively. Simulation results are presented for varying number of ZigBee nodes, varying number of WLAN nodes, and different packet arrival rates of WLAN and ZigBee nodes. We refer 
Table 1 MAC parameters of IEEE 802.11 b and IEEE 802.15.4 network

\begin{tabular}{lll}
\hline Parameter & $802.11 \mathrm{~b}$ & 802.15 .4 \\
\hline Data rate & $11 \mathrm{Mbps}$ & $250 \mathrm{Kpbs}$ \\
Slot time & $\delta=20 \mu \mathrm{s}$ & $t_{\mathrm{bo}}=320 \mu \mathrm{s}$ \\
SIFS & $30 \mu \mathrm{s}$ & $192 \mu \mathrm{s}$ \\
DIFS & $50 \mu \mathrm{s}$ & $\mathrm{N} / \mathrm{A}$ \\
CCA & $\mathrm{N} / \mathrm{A}$ & $128 \mu \mathrm{s}$ \\
MAC header & $24 \mathrm{~B}$ & $7 \mathrm{~B}$ \\
PHY header & $16 \mathrm{~B}$ & $1 \mathrm{~B}$ \\
Payload size & $1024 \mathrm{~B}$ & $120 \mathrm{~B}$ \\
ACK & $14 \mathrm{~B}$ & $11 \mathrm{~B}$ \\
ACK timeout & $300 \mu \mathrm{s}$ & $1120 \mu \mathrm{s}$ \\
CW min & 32 & $\mathrm{~N} / \mathrm{A}$ \\
\hline
\end{tabular}

N/A, not available

to the symmetric and asymmetric scenarios as scenarios 1 and 2 in the following.

To obtain numerical results, we have developed an event-driven simulator on Matlab where we have implemented all MAC protocol functions (randome backoff, CCA, data transmission, ACK, DIFS, and SIFS) for both WLAN and WPAN exactly with the corresponding time intervals. In particular, the backoff slots of the WLAN and WPAN nodes are $\delta=20 \mu \mathrm{s}$ and $t_{\mathrm{bo}}=320 \mu \mathrm{s}$, respectively. Therefore, the WPAN nodes are less responsive to accessing the channel compared to WLAN nodes. In addition, we have implemented the corresponding carrier sensing outcomes for the considered symmetric and asymmetric scenarios. Specifically, the WLAN and WPAN nodes can sense the transmissions from each other in the symmetric scenario while the WPAN nodes can sense the transmissions from WLAN nodes but not vice versa in the asymmetric scenario. Moreover, to obtain reliable throughput values for each considered setting, we have run both MAC protocols of the two coexisting networks over sufficiently long simulation time and calculate the achievable throughput along the way. Each simulation run is stopped only if the achievable throughput changes less than $1 \%$ around its stable value. Moreover, each point in the throughput curves of each figure is obtained through one independent simulation run described above. For better presentation, we do not show simulation reliability parameters such as standard deviations in all the figures. Moreover, we have assumed that both WLAN and WPAN employ the fixed transmission rates. Therefore, the link adaptation with multiple rates to exploit the time-varying wireless channel is not considered.

In Fig. 4, we show the throughput of ZigBee network versus the packet arrival rate of WLAN nodes $\left(\lambda_{\mathrm{W}}\right)$ for $n_{\mathrm{w}}=5,10, n_{\mathrm{z}}=5$, and two values of packet arrival rate of ZigBee nodes $\lambda_{\mathrm{z}}=10,50 \mathrm{pkt} / \mathrm{s}$ for scenario 1 . The figure

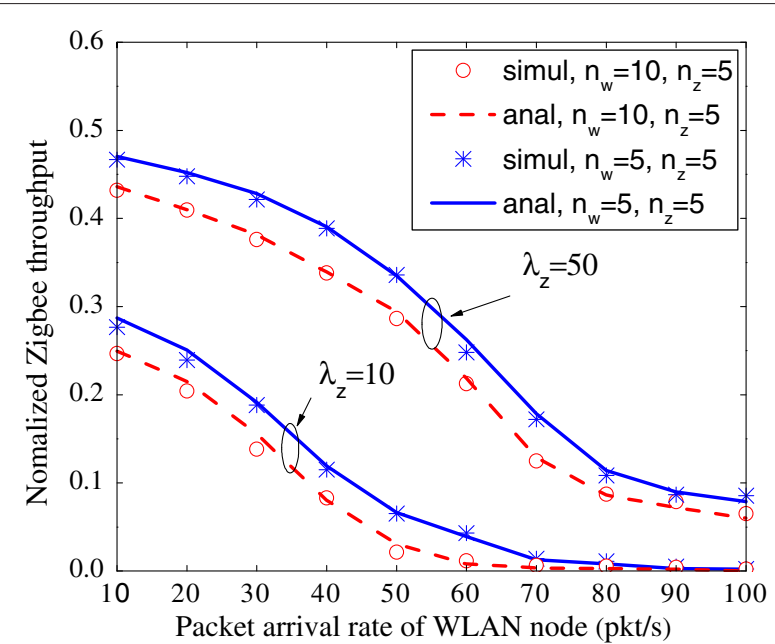

Fig. 4 Throughput of 802.15 .4 network in coexistence between 802.15.4 and unsaturated 802.11 networks in scenario 1

confirms that the throughput achieved by the proposed analytical model matches with the simulation results very well, which validate our model. The figure also shows that the ZigBee throughput decreases when the packet arrival rate of WLAN node increases. Moreover, larger number of WLAN node results in lower throughput for the ZigBee network. Moreover, the ZigBee throughput tends to zero as the WLAN node's load reaches the saturated regime.

In Fig. 5, we show that as the number of ZigBee nodes increases, its throughput decreases as expected. In addition, if there is no WLAN nodes coexisting with ZigBee nodes, the ZigBee throughput is much higher than that under the coexistence in scenario 1 . This is because WLAN nodes can prevent the channel access from ZigBee nodes. In fact, the ZigBee backoff slot duration is much

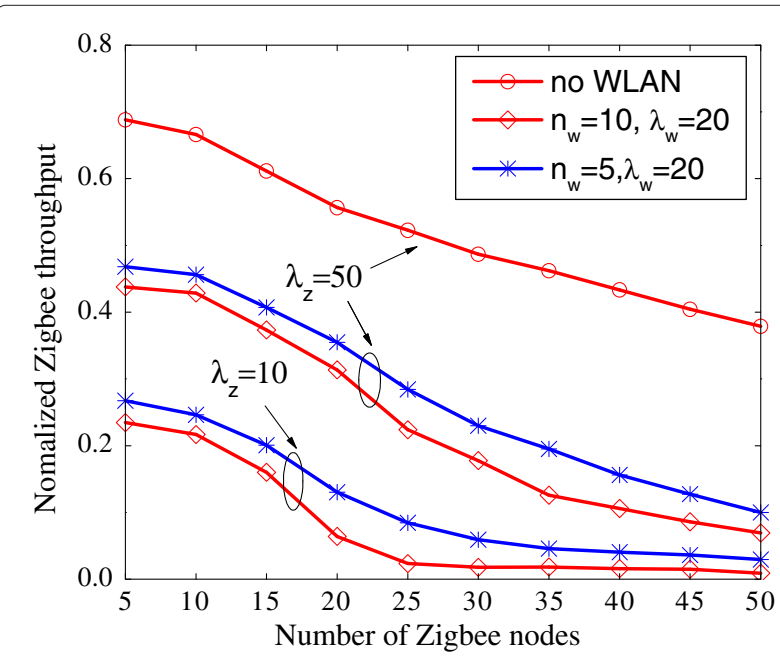

Fig. 5 Throughput of 802.15.4 network versus number of ZigBee nodes in scenario 1 
longer than the WLAN backoff slot duration, which limits the ZigBee nodes' opportunities to access the channel in comparison with WLAN nodes. The ZigBee throughput performance also varies as we vary the number of WLAN nodes and the packet arrival rate of WLAN nodes as well as the packet arrival rate of ZigBee nodes. Specifically, larger number of WLAN nodes and/or smaller packet arrival rate of ZigBee nodes result in lower throughput of the ZigBee network. Figure 6 demonstrates that when the packet arrival rate of ZigBee node increases, its throughput increases in scenario 1 . However, when the ZigBee packet arrival rate reaches the saturation value, the throughput decreases slightly. In fact, with higher ZigBee packet arrival rate, the busy probability in CCAs become higher because of more transmitted packets and WLAN preemption. ${ }^{4}$

In Fig. 7, we validate the accuracy of the proposed analytical model for scenario 2 . The figure shows that, in the presence of WLAN interference, the ZigBee throughput decreases very rapidly down to about 0.025 for ZigBee packet arrival rate $\lambda_{\mathrm{z}}=50$ and zero for $\lambda_{\mathrm{z}}=10$. Similar to scenario 1 , when the WLAN packet arrival rate increases, the ZigBee throughput decreases dramatically. In scenario 2, the ZigBee throughput is lower and decreases faster than that in scenario 1 . This is because, in scenario 2, WLAN nodes cause more collisions to ZigBee transmissions since WLAN nodes cannot sense and detect the ZigBee transmissions. Figure 8 depicts the ZigBee throughput for varying number of ZigBee nodes $n_{\mathrm{Z}}$ in scenario 2 . This figure shows that the ZigBee throughput tends to zero as the number of ZigBee nodes becomes larger.

In Fig. 9, we illustrate the ZigBee throughput versus the ZigBee packet arrival rate. This figure confirms that the ZigBee throughput increases as the ZigBee packet arrival

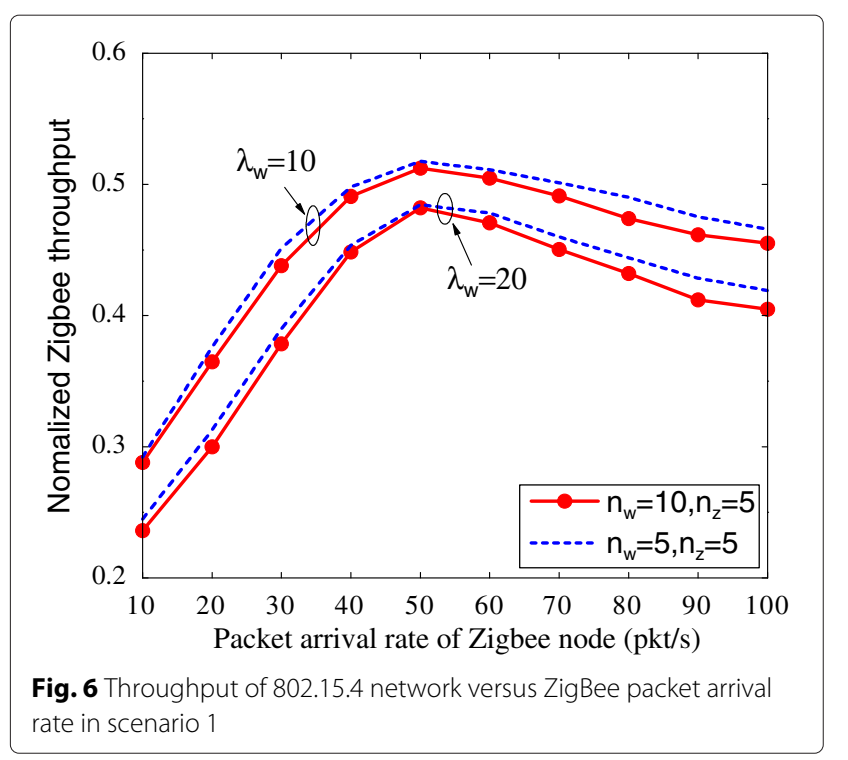

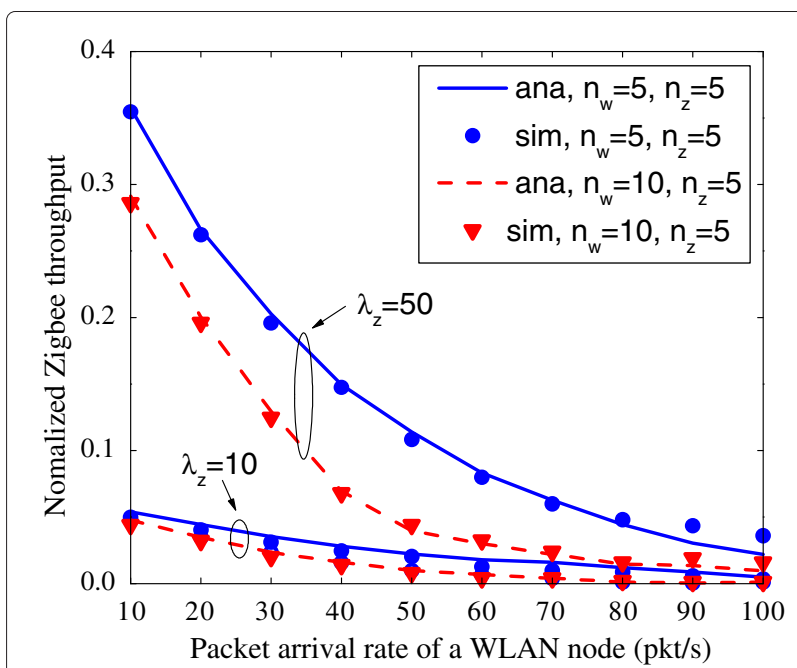

Fig. 7 Throughput of 802.15.4 network versus WLAN packet arrival rate in the scenario 2

rate increases. However, when the ZigBee packet arrival rate becomes sufficiently large, the ZigBee throughput decreases slightly. This is due to the fact that the busy probabilities in two CCAs are larger as the ZigBee packet arrival rate reaches the saturated traffic condition. In addition, the WLAN preemption and ZigBee collisions due to WLAN transmissions happen more frequently in scenario 2. Therefore, the ZigBee throughput in scenario 2 is much lower than that in scenario 1. In Fig. 10, we demonstrate the WLAN throughput versus the WLAN packet arrival rate. It can be observed that the WLAN throughput increases and then slightly decreases as the WLAN packet arrival rate becomes larger. Moreover, when $\lambda_{\mathrm{w}}$ is less than a critical value around $70 \mathrm{pkt} / \mathrm{s}$, the WLAN throughput in the scenario 1 is higher than that in the scenario 2 and

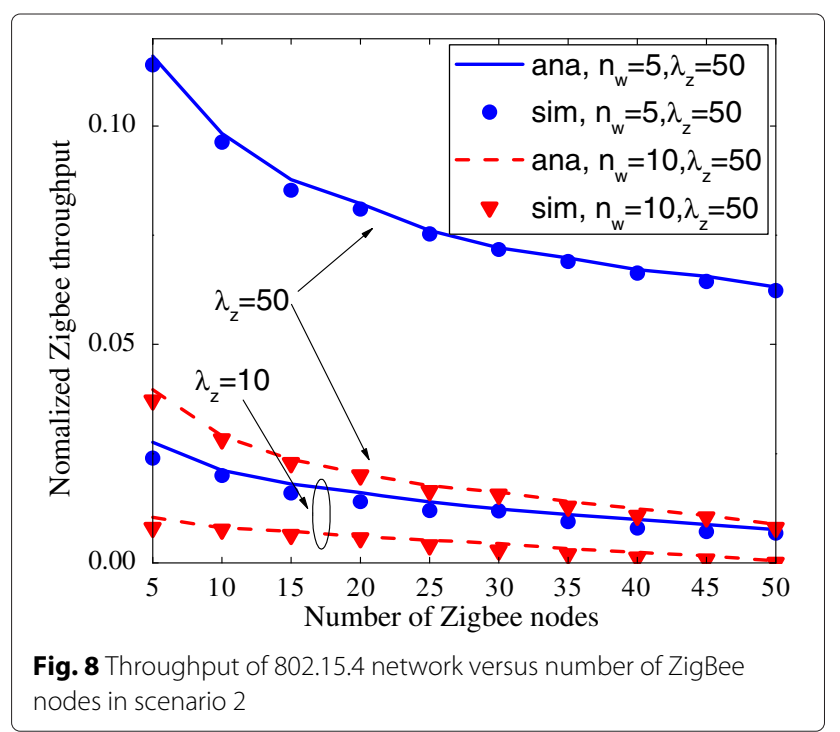




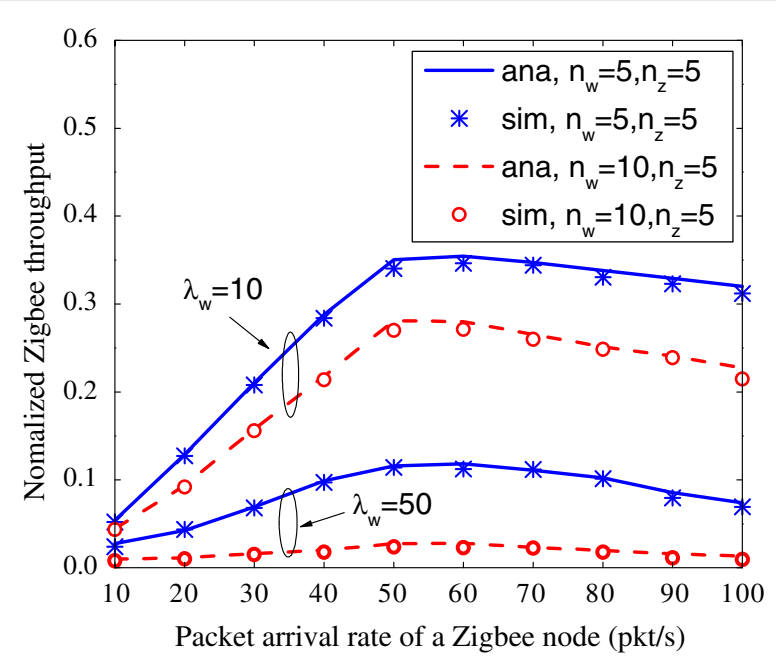

Fig. 9 Throughput of 802.15.4 network versus ZigBee packet arrival rate in scenario 2

the reverse is true if $\lambda_{\mathrm{w}}$ is larger than the critical WLAN arrival rate.

Finally, we illustrate the variation of $\Delta S\left(n_{1}, n_{2}\right)$, which is the objective of problem (53), for different channel assignment solutions in Fig. 11 considering the actual channel deployment for ZigBee and WLAN [1] where $\left|\Omega_{\text {over }}\right|=$ $12,\left|\Omega_{\text {non }}\right|=4$. We set $n_{\mathrm{z}}$ equal to 70 and 120 for scenarios 1 and 2, respectively. Here, $n_{\mathrm{w}}$ is set to 10 in every overlapping channels, and $\lambda_{\mathrm{z}}$ and $\lambda_{\mathrm{z}}$ are equal to $50 \mathrm{pkt} / \mathrm{s}$. Figure 11 also shows that $\left(n_{1}, n_{2}\right)=(2,12)$ results in the fairest throughput sharing for ZigBee nodes in scenario 1 while $\left(n_{1}, n_{2}\right)=(1,27)$ achieves fairest throughput sharing for scenario 2 .

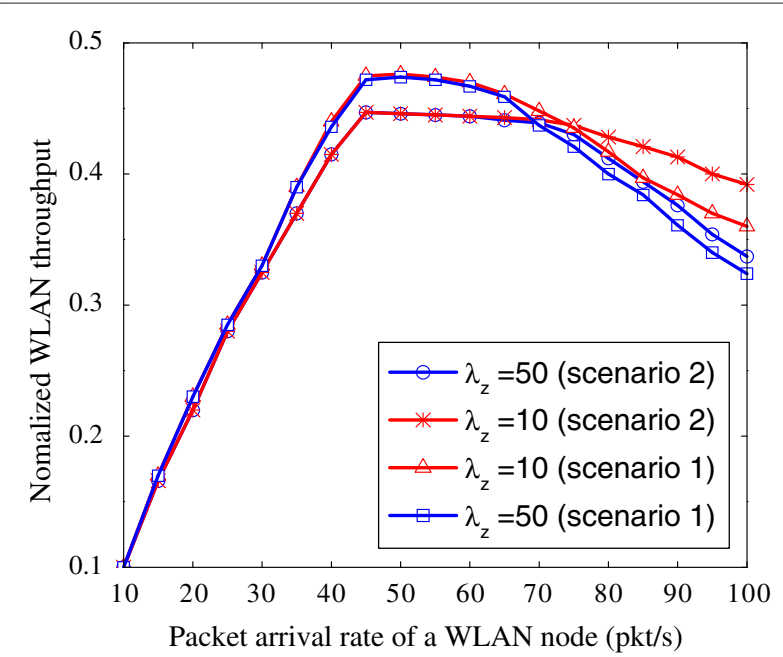

Fig. 10 Throughput of WLAN versus WLAN packet arrival rate for number of WLAN $n_{\mathrm{w}}=10$ and number of ZigBee network $n_{z}=10$ in both scenario 1 and scenario 2

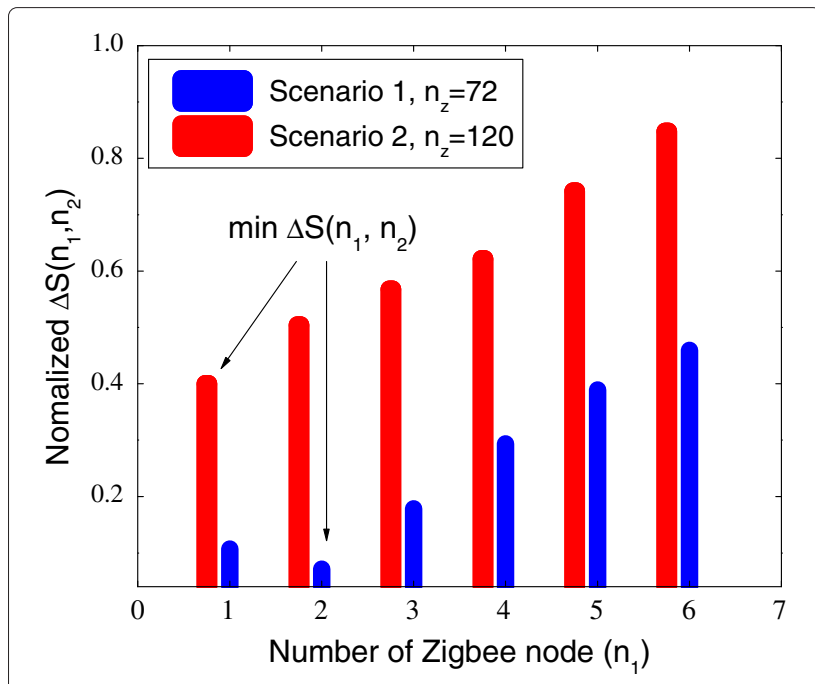

Fig. 11 Variation of $\Delta S\left(n_{1}, n_{2}\right)$ under different channel allocation solutions

\section{Conclusions}

We have developed analytical models for throughput evaluation of ZigBee nodes coexisting with WLAN nodes in the IEEE 802.11 network in both symmetric and asymmetric scenarios. The proposed models are based on the $\mathrm{MC}$ analysis for one pair of typical (WLAN, ZigBee) nodes considering detailed operations of MAC protocols in the two networks. We have then proposed to employ the analytical model for channel allocation that achieves fair throughput sharing among ZigBee nodes. We have validated the analytical models through simulation studies. The impacts of different parameters on the throughput performance of the ZigBee network and WLAN have been also studied.

\section{Endnotes}

${ }^{1}$ In this paper, we adopt the following notation $P\left\{y_{2}, z_{2} \mid y_{1}, z_{1}\right\}=P\left\{a(t+1)=y_{2}, b(t+1)=z_{2} \mid a(t)=\right.$ $\left.y_{1}, b(t)=z_{1}\right\}$

${ }^{2}$ The "generic slot" concept has been proposed by Bianchi in his seminal paper on the performance analysis of the CSMA/CA MAC protocol for the 802.11 network [15]. A generic slot indeed captures different possible "slot" or intervals related to the operations of the underlying MAC protocol, namely the backoff slot, the transmission interval during which the data packet is transmitted.

${ }^{3}$ If the arrival rates of WLAN on different channels and the number of WLAN nodes $n_{\mathrm{W}}$ on each overlapping channel are different, then we have to optimize multiple variables $n_{i}$, which is the number of ZigBee nodes assigned for channel $i$. Therefore, it is still possible even though the optimization complexity is higher. 
${ }^{4}$ The ZigBee network can become unstable for sufficiently large values of the arrival rate of the ZigBee node. Note that for the "unstable" regime, the network will be in the saturated traffic regime where each node always has data to transmit.

\section{Competing interests}

The authors declare that they have no competing interests.

\section{Acknowledgements}

This work was supported by the Natural Sciences and Engineering Research Council (NSERC) of Canada under the Strategic Partnership Grant (SPG), UM Project\# 40247.

Received: 17 April 2015 Accepted: 13 March 2016

Published online: 06 May 2016

\section{References}

1. C-JM Liang, NB Priyantha, J Liu, A Terzis, in Proc. SenSys'10. Surviving Wi-Fi interference in low power ZigBee networks, (NewYork, 2010), pp. 309-322

2. W Yuan, X Wang, J-PMG Linnartz, in Proc. IEEE VTC. A coexistence model of IEEE 802.15.4 and IEEE 802.11 b/g, (Delft, Netherlands, 2007), pp. 1-5

3. S Pollin, I Tan, B Hodge, C Chun, A Bahai, in Proc. CrownCom. Harmful coexistence between 802.15.4 and 802.11: a measurement-based study, (Singapore, 2008), pp. 1-6

4. A Sikora, VF Groza, in Proc. IEEE IMTC 2005. Coexistence of IEEE 802.15.4 with other systems in the $2.4 \mathrm{GHz}-I S M-B a n d$, (Ottawa, 2005), pp. 1786-1791

5. W Yuan, X Wang, J-PMG Linnartz, IGMM Niemegeers, Coexistence performance of IEEE 802.15.4 wireless sensor networks under IEEE $802.11 \mathrm{~b} / \mathrm{g}$ interference. Wirel. Pers. Commun. 68(2), 281-302 (2013)

6. I Howitt, JA Gutierrez, in Proc. IEEE WCNC. IEEE 802.15.4 low rate-wireless personal area network coexistence issues, vol. 3, (LA, USA, 2003), pp. 1481-1486

7. L Angrisani, M Bertocco, D Fortin, A Sona, Experimental study of coexistence issues between IEEE $802.11 \mathrm{~b}$ and IEEE 802.15.4 wireless networks. IEEE Trans. Instrum. Meas. 57(8), 1514-1523 (2008)

8. R de Francisco, L Huang, G Dolmans, in Proc. IEEE VTC. Coexistence of WBAN and WLAN in medical environments, (Anchorage, AK, US, 2009), pp. 1-5

9. X Zhang, KG Shin, Cooperative carrier signaling: harmonizing coexisting WPAN and WLAN devices. IEEE/ACM Trans. Netw. 21(2), 426-439 (2013)

10. $X$ Zhang, KG Shin, in Proc. ACM MobiHoc. Enabling coexistence of heterogeneous wireless systems: case for ZigBee and WiFi, (2011)

11. J Huang, G Xing, G Zhou, R Zhou, in Proc. IEEE ICNP 2010. Beyond co-existence: exploiting WiFi white space for ZigBee performance assurance, (Kyoto, 2010), pp. 305-314

12. S Geirhofer, L Tong, BM Sadler, Dynamic spectrum access in the time domain: modeling and exploiting white space. IEEE Commun. Mag. 45(5), 66-72 (2007)

13. P Yi, A Iwayemi, C Zhou, Developing ZigBee deployment guideline under WiFi interference for smart grid applications. IEEE Trans. Smart Grid. 2(1), 110-120 (2011)

14. J-S Han, S-H Lee, H-S Kim, Y-H Lee, in Proc. IEEE WCNC 2011. Performance improvement of IEEE 802.15.4 in the presence of co-channel interference, (Cancun, Quintana Roo, 2011)

15. G Bianchi, Performance analysis of the IEEE 802.11 distributed coordination function. IEEE J. Sel. Areas Commun. 18(3), 535-547 (2000)

16. F Cali, M Conti, E Gregori, Dynamic tuning of the IEEE 802.11 protocol to achieve a theoretical throughput limit. IEEE/ACM Trans. Netw. 8(6), 785-799 (2000)

17. F Daneshgaran, M Laddomada, F Mesiti, M Mondin, Unsaturated throughput analysis of IEEE 802.11 in presence of non ideal transmission channel and capture effects. IEEE Trans. Wirel. Comm. 7(4), 1276-1286 (2008)

18. I Ramachandran, AK Das, S Roy, Analysis of the contention access period of IEEE 802.15.4 MAC. ACM Trans. Sens. Netw. 3(4), 1-29 (2007)
19. X Ling, Y Cheng, JW Mark, X Shen, A renewal theory based analytical model for the contention access period of IEEE 802.15.4 MAC. IEEE Trans. Wirel. Comm. 7(6), 2340-2349 (2008)

20. CY Jung, HY Hwang, DK Sung, GU Hwang, Enhanced Markov chain model and throughput analysis of the slotted CSMA/CA for IEEE 802.15.4 under unsaturated traffic conditions. IEEE Trans. Veh. Tech. 58(1), 473-478 (2009)

21. S Pollin, M Ergen, SC Ergen, B Bougard, LVd Perre, I Moerman, A Bahai, P Varaiya, $F$ Catthoor, Performance analysis of slotted carrier sense IEEE 802.15.4 medium access layer. IEEE Trans. Wirel. Commun. 7(9) (2008)

22. SY Shin, HS Park, WH Kwon, Mutual interference analysis of IEEE 802.15.4 and IEEE 802.11b. Comput. Netw. 51(12), 3338-3353 (2007)

23. IEEE 802.15.4 Specification, Wireless Medium Access Control (MAC) and Physical Layer (PHY) Specifications for Low-Rate Wireless Personal Area Networks (LR-WPANS), (2003)

24. Wireless LAN Medium Access Control (MAC) and Physical Layer (PHY) Specifications, IEEE standards 802.11 (1997)

25. G Thonet, P Allard-Jacquin, P Colle, ZigBee WiFi coexistence, white paper and test report, Schneider Electric (2008)

26. P Luong, TM Nguyen, LB Le, Throughput analysis for coexisting IEEE 802.15.4 and 802.11 networks under unsaturated traffic. Technical report. Online: http://www.necphy-lab.com/pub/CoexistenceReport.pdf

\section{Submit your manuscript to a SpringerOpen ${ }^{\circ}$ journal and benefit from:}

- Convenient online submission

- Rigorous peer review

- Immediate publication on acceptance

- Open access: articles freely available online

- High visibility within the field

- Retaining the copyright to your article

Submit your next manuscript at $\mathbf{s p r i n g e r o p e n . c o m ~}$ 